\title{
Separating melanin from hemodynamics in nevi using multimode hyperspectral dermoscopy and spatial frequency domain spectroscopy
}

Fartash Vasefi

Nicholas MacKinnon

Rolf Saager

Kristen M. Kelly

Tyler Maly

Nicholas Booth

Anthony J. Durkin

Daniel L. Farkas 


\title{
Separating melanin from hemodynamics in nevi using multimode hyperspectral dermoscopy and spatial frequency domain spectroscopy
}

\author{
Fartash Vasefi, ${ }^{\mathrm{a}, \star}$ Nicholas MacKinnon, ${ }^{\mathrm{a}}$ Rolf Saager, ${ }^{\mathrm{b}}$ Kristen M. Kelly, ${ }^{\mathrm{b}}$ Tyler Maly, ${ }^{\mathrm{b}}$ Nicholas Booth, ${ }^{\mathrm{a}}$ \\ Anthony J. Durkin, ${ }^{b}$ and Daniel L. Farkas ${ }^{a, c, *}$ \\ aspectral Molecular Imaging Inc., 13412 Ventura Boulevard, Suite 250, Sherman Oaks, California 91423, United States \\ bUniversity of California, Beckman Laser Institute and Medical Clinic, 1002 Health Sciences Road, Irvine, California 92612, United States \\ 'University of Southern California, Department of Biomedical Engineering, 1042 Downey Way, Los Angeles, California 90089, United States
}

\begin{abstract}
Changes in the pattern and distribution of both melanocytes (pigment producing) and vasculature (hemoglobin containing) are important in distinguishing melanocytic proliferations. The ability to accurately measure melanin distribution at different depths and to distinguish it from hemoglobin is clearly important when assessing pigmented lesions (benign versus malignant). We have developed a multimode hyperspectral dermoscope (SkinSpect ${ }^{\mathrm{TM}}$ ) able to more accurately image both melanin and hemoglobin distribution in skin. SkinSpect uses both hyperspectral and polarization-sensitive measurements. SkinSpect's higher accuracy has been obtained by correcting for the effect of melanin absorption on hemoglobin absorption in measurements of melanocytic nevi. In vivo human skin pigmented nevi $(N=20)$ were evaluated with the SkinSpect, and measured melanin and hemoglobin concentrations were compared with spatial frequency domain spectroscopy (SFDS) measurements. We confirm that both systems show low correlation of hemoglobin concentrations with regions containing different melanin concentrations ( $R=0.13$ for SFDS, $R=0.07$ for SkinSpect). $\odot 2016$ Society of Photo-Optical Instrumentation Engineers (SPIE) [DOI: 10.1117/1.JBO.21.11.114001]
\end{abstract}

Keywords: hyperspectral imaging; polarization; melanoma; tissue phantoms; spatial frequency domain; melanin.

Paper 160457SSPR received Jul. 1, 2016; accepted for publication Oct. 7, 2016; published online Nov. 10, 2016.

\section{Introduction}

The incidence of skin cancer has reached epidemic levels, and melanoma is of particular concern. An estimated 76,380 new cases of invasive melanoma will be diagnosed in the United States in 2016. An estimated 10,130 people will die of melanoma in 2016. Melanoma accounts for $<1 \%$ of skin cancer cases but the vast majority of skin cancer deaths. ${ }^{1,2}$ A recent study demonstrated that the health and economic burden of skin cancer treatment is substantial and, in the United States, increased from $\$ 3.6$ billion in 2002-2006 to $\$ 8.1$ billion in 2007-2011. ${ }^{3}$ Noninvasive detection of melanoma, especially early in the evolution of this deadly disease, is extremely important for outcomes: most lesions caught early and removed surgically yield a likelihood of cancer eradication of at least $86 \%$ (10-year survival rate). In contrast to this, late detection leads to very high $(>90 \%)$ mortality rates (10-year survival rate between $10 \%$ and $15 \%) .{ }^{4}$ Immunotherapies are becoming the standard of care for metastatic melanoma. They are quite effective but are currently extremely costly and can be in excess of $\$ 250,000$. $^{5}$

Noninvasive optical imaging offers an important opportunity for early detection of skin cancer. Spectroscopic methods can be used to quantify tissue-specific variables such as oxygenation and melanin distribution at the molecular level and aid noninvasive diagnosis. However, current commercial and research systems used for mapping of hemoglobin and melanin in skin do not provide accurate information when applied to pigmented

*Address all correspondence to: Fartash Vasefi, E-mail: fvasefi@gmail.com; Daniel L. Farkas, E-mail: dlfarkas@gmail.com lesions and different skin types. Prior studies by other groups have demonstrated difficulty distinguishing melanin from hemoglobin. ${ }^{6-8}$ The main source of melanin-hemoglobin crosstalk error is spectral absorption overlap of deoxy-hemoglobin and melanin, resulting in high melanin content being misestimated as high deoxy-hemoglobin content and leading to higher total hemoglobin concentration and lower oxygen saturation being reported in pigmented regions. ${ }^{6}$ Another source of misestimation is due to insufficient discrete wavelength measurements or using only the color camera Bayer filter for spectral selection. ${ }^{9}$ More accurate discrimination between melanin and hemoglobin spectral signatures is important for determining the distribution of melanin and hemoglobin and particularly the depth distribution of melanin. For example, dermatologists are using a seven-point checklist including atypical pigmented distribution as well as atypical vascular distribution during melanoma assessment. ${ }^{10}$ In our previous studies, we have discussed the nature of these errors and how our algorithms using multimode hyperspectral measurements can overcome this melaninhemoglobin absorption crosstalk. ${ }^{11,12}$ Changes in the pattern and distribution of both melanocytes (pigment producing) and vasculature (hemoglobin containing) are important in distinguishing melanocytic proliferations. Melanomas tend to be more vascular than blue nevi, congenital nevi, and acquired melanocytic nevi. ${ }^{13-16}$ In melanomas, the degree of vascularity is positively correlated with Breslow's depth (a measure of tumor thickness ${ }^{17,18}$ ), which is an important prognostic factor. ${ }^{19}$ The ability to accurately measure melanin distribution at different

$1083-3668 / 2016 / \$ 25.00$ @ 2016 SPIE 


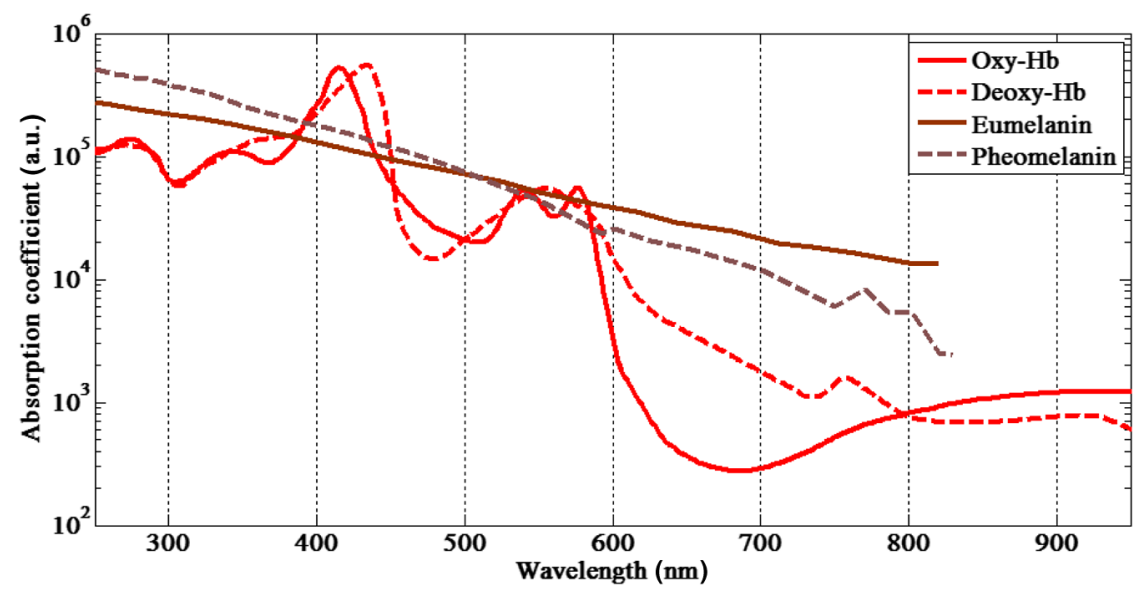

Fig. 1 The absorption spectra of melanin and hemoglobin. ${ }^{28,29,30}$

depths and to distinguish it from hemoglobin is clearly important to quantify the progress of melanocyte invasion.

We have recently introduced a new multimode dermoscope called SkinSpect (Spectral Molecular Imaging, Beverly Hills, California) that combines polarization and hyperspectral imaging to accurately map the distribution of skin melanin and hemoglobin. Ultimately, we hope to develop this technology to a sufficient degree that it can be used to improve skin cancer screening. Initial studies ${ }^{11}$ were limited by the small number of patients and lack of comparative analysis to other widely accepted skin assessment devices to validate the mapping accuracy of SkinSpect.

In the next section, we briefly review several aspects related to light propagation in tissue to illustrate the strengths and limitations of the optical techniques employed here.

\subsection{Skin Optics}

Information about skin physiology, morphology, and composition can be obtained noninvasively by optical imaging methods. Photons propagating through tissue can be scattered by collagen fibers or by membranes because of refractive index fluctuations at a microscopic level, ${ }^{20}$ have their polarization state altered following multiple scattering events, or be absorbed by molecules such as hemoglobin or melanin. Skin has a compound layer structure comprising dermal and epidermal layers. Normal epidermis thickness ranges between $\sim 30$ and $150 \mu \mathrm{m} .{ }^{21,22}$ Melanin is found in the epidermis in the form of red/yellow pheomelanin and/or brown/black eumelanin that absorbs very broadly in the visible spectrum with higher values for shorter wavelengths $(<600 \mathrm{~nm})$. Human skin color is characterized by variable concentration of eumelanin, where the volume fraction of the epidermis occupied by melanosomes varies and can range from $1.3 \%$ [light Caucasian skin (type I)] to $43 \%$ [very dark in black African skin (type VI)]. ${ }^{23}$ The dermis thickness can vary from $0.6 \mu \mathrm{m}$ to $3 \mathrm{~mm}$ (Ref. 24) and consists of the papillary dermis and reticular dermis. The dermis is composed of connective tissues, blood vessels, and nerves. Blood concentration in dermis varies from $0.2 \%$ to $7 \%$ in volume fraction. ${ }^{23}$ In the blood cells, there are several natural chromophores, primarily hemoglobin, which gives blood its reddish color. The hemoglobin oxygenation varies from $47 \%$ in veins to $90 \%$ to $95 \%$ in arteries. ${ }^{25}$ The total hemoglobin concentration in blood is between 134 and $173 \mathrm{~g} / \mathrm{L} .{ }^{26}$ Hemoglobin is found mainly in its oxy- and deoxy-hemoglobin forms in the microvascular network of the dermis, normally 50 to $500 \mu \mathrm{m}$ below the skin surface. ${ }^{27}$ Other skin chromophores can include bilirubin and $\beta$-carotene that, when found in the dermis, contributes to the yellowish or olive tint of human skin. These are not major contributors in pigmented nevi. Figure 1 illustrates the absorption spectra of skin constituents that are studied in this work. The melanin and hemoglobin absorptions are orders of magnitude stronger and are the major contributors to skin absorption.

Melanin fraction, epidermal thickness, and hemoglobin concentration all affect skin absorption spectra and interact with each other in complex ways that affect skin measurements from different anatomical regions and different skin types. To illustrate this complexity, in Fig. 2, we have used the biophysically based spectral model of light interaction with human skin (BioSpec) modeling software ${ }^{28}$ developed by researchers at University of Waterloo, Canada. We used this modeling system because it lets us easily vary skin parameters using a web-based platform to demonstrate how epidermal thickness and skin types can affect skin absorption spectra. The spectral input data files used by the online BioSpec model are available in Ref. 28 and are compiled from skin chromophore spectral libraries from well-known researchers. ${ }^{29,30}$

Figure 2(a) shows the skin absorption spectra variation resulting from changing the percentage of epidermis occupied by melanosomes from $1 \%$ to $5 \%$. Figure 2(b) shows the skin spectral absorption change due to changing the concentration of hemoglobin in the blood from 100 to $200 \mathrm{~g} / \mathrm{L}$. Figure 2(c) shows the skin absorption spectra variation resulting from varying the epidermal thickness from 50 to $150 \mu \mathrm{m}$. In this figure, as one of the three parameters varies, the other two parameters remain constant. The values held constant are the percentage of epidermis occupied by melanosome (1.3\%), epidermal thickness $(100 \mu \mathrm{m})$, and concentration of hemoglobin in the blood (147 g/L). Figure 2(d) shows the complexity of absorption spectra resulting from changing any of the three skin parameters. As can be seen, different combinations of melanin, hemoglobin, or epidermal thickness values can have similar absorption spectra patterns. We have developed SkinSpect to use the depth-sensitive techniques of hyperspectral imaging and polarization imaging to disentangle the complexities associated with varying combinations of melanin, hemoglobin, and the effect of epidermal thickness. 
(a)
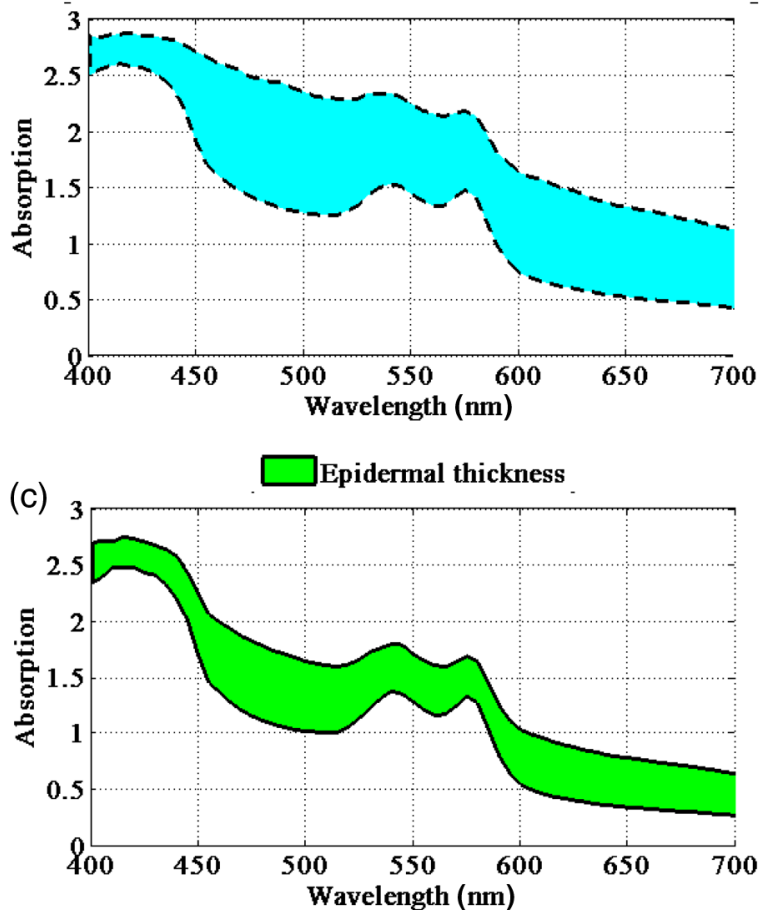

(b) Concentration of hemoglobin in the blood

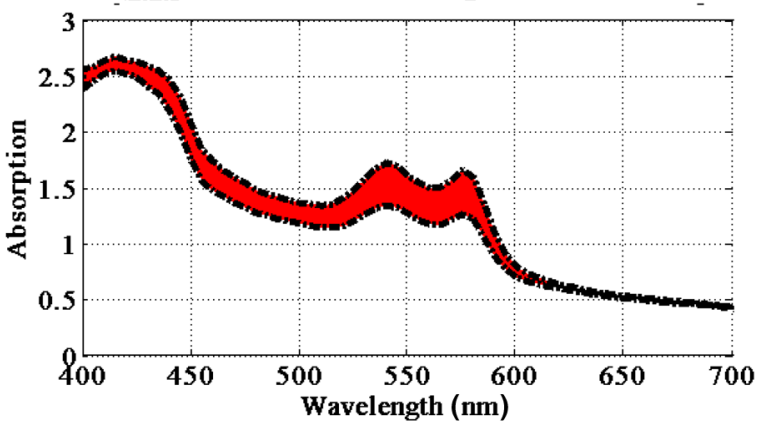

(d)

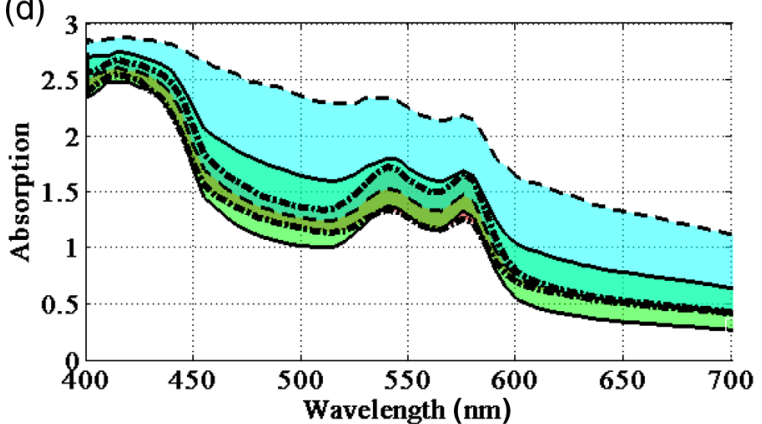

Fig. 2 Skin absorption spectra at various epidermal thicknesses and melanin and hemoglobin concentrations: (a) variations at the percentage of epidermis occupied by melanosome ( $1 \%$ to $5 \%)$, (b) variations on concentration of hemoglobin in the blood (100 to $200 \mathrm{~g} / \mathrm{L}$ ), (c) epidermal thickness variations (50 to $150 \mu \mathrm{m}$ ), and (d) overlapping skin absorption spectral regions at all three skin properties variations described at (a)-(c).

\subsection{SkinSpect}

We are addressing this issue by developing methods for quantitative analysis of skin using our multimode hyperspectral dermoscope. The key advantage of this multimode optical imaging technology is to provide biologically plausible measurements of skin at the molecular level (melanin, hemoglobin) and depth-resolved spatial distribution of skin features. SkinSpect software is able to use image datacubes from parallel and cross-polarized measurements to more accurately estimate melanin and hemoglobin. ${ }^{11,12}$

\subsection{Spatial Frequency Domain Spectroscopy}

We compare melanin and hemoglobin measurements from SkinSpect to those obtained using spatial frequency domain spectroscopy (SFDS) [also known as spatially modulated quantitative spectroscopy (SMoQS)]. We chose SFDS as a validation method because it is also an approach that is based in biological plausibility, employs high spectral resolution, and separates melanin and hemoglobin contributions effectively. To validate the accuracy of SFDS's two-layer model approach, Saager et al. have compared SFDS in vivo skin data to data obtained from multiphoton microscopy using selective two-photon excited fluorescence of melanin. ${ }^{31}$ Subjects ranged in pigmentation from very light skin to dark skin (Fitzpatrick types I to VI). ${ }^{32}$ Using the depth-sectioning capabilities of multiphoton microscopy as a reference, a layered model interpretation of SFDS data was able to demonstrate a linear correlation for in vivo average concentration and depth distribution of melanin to within $\sim 15 \%$ and tens of $\mu \mathrm{m}$, respectively.
In a separate study, SFDS was also able to demonstrate that this two-layer model approach was able to significantly decouple and isolate melanin from induced hemodynamic events in healthy subjects $(n=9)$ across a distribution of skin pigmentation. ${ }^{33}$ This study not only illustrated how interpretation of results using a layer model can be insensitive to crosstalk errors induced by depth-specific sources of optical contrast (i.e., melanin and hemoglobin), but also demonstrated how significantly these errors can affect the estimation of underlying hemoglobin concentrations when high concentrations of melanin might be present (e.g., pigmented lesions).

\section{Methods and Materials}

\subsection{SkinSpect System}

\subsubsection{SkinSpect instrument}

SkinSpect illuminates human skin under study with a spectrally programmable linearly polarized light source at 33 wavelengths $(\mathrm{FWHM}=22 \mathrm{~nm}$ ) equally spaced between 468 and $857 \mathrm{~nm}$. This range was selected to encompass the main absorption features of melanin and oxy- and deoxy-hemoglobin including isosbestic points in visible and near-infrared (NIR) regions. ${ }^{34}$ Diffusely reflected photons are separated into parallel and cross-polarized image paths and images captured for each illumination wavelength (see Fig. 3). We have developed a method that combines two depth-sensitive techniques, polarization and hyperspectral imaging, to accurately determine the spatial distribution of melanin and hemoglobin oxygenation in a skin lesion. As shown in Fig. 3, SkinSpect is able to image the fluorescence emission of skin (primarily from collagen); however, in 


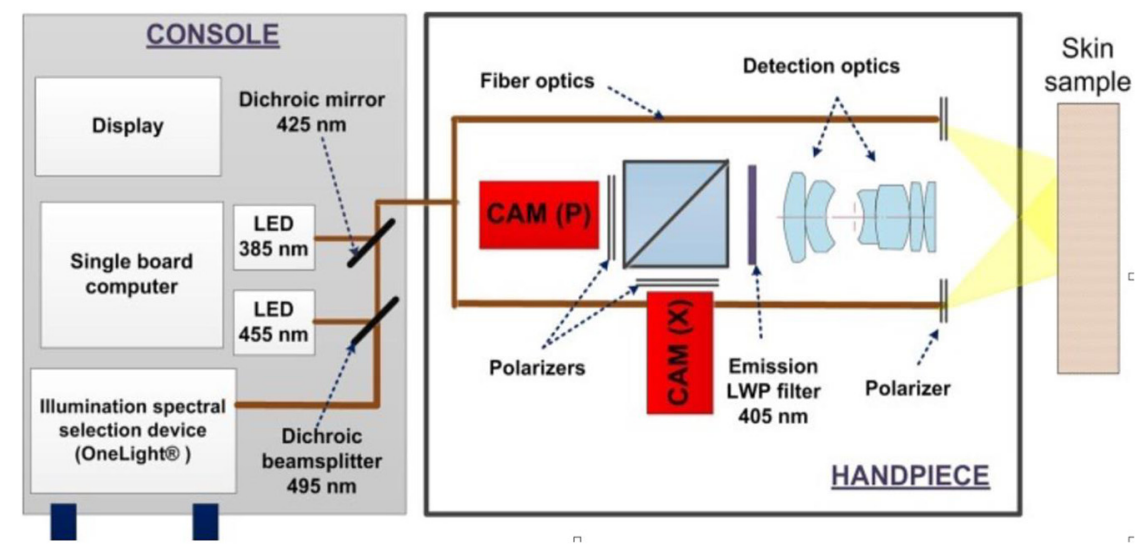

Fig. 3 SkinSpect system diagram with dual camera hand-piece configuration.

this paper, we limit our analysis to polarization and hyperspectral imaging to study skin melanin and hemodynamics. SkinSpect's field of view is $18 \mathrm{~mm}$, which can spatially resolve features down to $\sim 100 \mu \mathrm{m}$ in size. More details about the system, skin analysis algorithm, and our method of correcting for melanin misestimation are described in our previous publications. $^{5,12}$

\subsubsection{SkinSpect data processing}

The measured skin image cubes in parallel and cross-polarization $\left[\left(I_{\|_{\text {skin }}}(x, y, \lambda), I_{\perp_{\text {skin }}}(x, y, \lambda)\right]\right.$ are normalized by measurements using the same scanning sequence (wavelength and exposure time) with a white calibration target, Spectralon $\left[\left(I_{\|_{\text {spectralon }}}(x, y, \lambda), I_{\perp_{\text {spectralon }}}(x, y, \lambda)\right]\right.$. The normalized reflection image datacubes in both parallel $\left(R_{\|}\right)$and perpendicular $\left(R_{\perp}\right)$ polarization states are computed using the following equation:

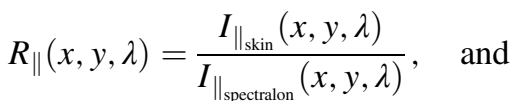

$R_{\perp}(x, y, \lambda)=\frac{I_{\perp_{\text {skin }}}(x, y, \lambda)}{I_{\perp_{\text {spectralon }}}(x, y, \lambda)}$.

Both $R_{\|}$and $R_{\perp}$ are affected by the superficial melanin that attenuates the reflectance. To remove the effect of superficial melanin attenuation to determine the chromophore absorption spectrum from deeper in the skin, we have introduced the polarization attenuation function $A_{\mathrm{POL}}$ as shown in the following equation:

$A_{\mathrm{POL}}=\log \left(\frac{R_{\|}-R_{\perp}}{R_{\|}}\right)$.

As described in our previous publication, ${ }^{11} A_{\mathrm{POL}}$ removes the absorption of the superficial layer, which contains predominately melanin, and isolates the absorption of deep melanin, oxy- and deoxy-hemoglobin. The slope of the $A_{\mathrm{POL}}$ function from 615 to $680 \mathrm{~nm}$ can be used as a measure of the relative concentration of deep melanin. At this wavelength range, melanin is less affected by the influence of hemoglobin absorption, as shown in measurements during venous occlusion in our previous study. ${ }^{11}$ The deep melanin spatial distribution $\operatorname{Mel}(x, y)$, can be estimated as
$\operatorname{Deep}-\operatorname{Mel}(x, y)=\operatorname{slope}\left\{A_{\mathrm{POL}(x, y, 615)}, A_{\mathrm{POL}(x, y, 680)}\right\}$.

We have used the cross-polarized absorption function to calculate total relative melanin distribution. Conventionally, an absorption function has a minus sign in the logarithmic function of reflectance; $A_{\perp}=-\log \left[R_{\perp}(x, y, \lambda)\right]$. Total melanin distribution is then calculated by

Total $-\operatorname{Mel}(x, y)=\operatorname{slope}\left\{A_{\perp(x, y, 615)}, A_{\perp(x, y, 680)}\right\}$.

To correct for melanin absorption, we subtract the relative absorption due to deep melanin to obtain the melanin-corrected $A_{\mathrm{POL}}$ function ( $\left.A_{\mathrm{POL}-\text { Melcorrected }}\right)$ which can be analyzed to determine the oxy- and deoxy-hemoglobin concentrations using the linear least-square regression analysis in the 500 to $577 \mathrm{~nm}$ wavelength range (seven wavebands). This range encompasses the local absorption spectrum maxima of both oxy- and deoxyhemoglobin. More details are described in our previous publications. $^{11,12}$

\subsection{Spatial Frequency Domain Spectroscopy}

\subsubsection{SFDS instrument}

SFDS is a depth-sensitive spectroscopy technique developed at the Beckman Laser Institute for quantitative in vivo measurement of tissue constituents including melanin, oxy- and deoxyhemoglobin, total hemoglobin, and oxygen saturation. ${ }^{35,31}$ SFDS collects depth-resolved reflectance information with $\sim 1 \mathrm{~nm}$ spectral resolution over the wavelength range 430 to $1050 \mathrm{~nm}$. Once collected, the frequency-dependent reflectance is transformed into the absorption coefficient and reduced scattering coefficient, ultimately with the goal of deducing skin chromophore concentrations. SFDS is a point-detection system with a field of view $1 \mathrm{~mm}$ in diameter. The implementation of SFDS in this particular study (Fig. 4) uses a 100-W quartz-tungsten halogen light source (Moritex, D100LR) for the broadband illumination source and a digital micromirror device (DMD; DLP developers kit, Texas Instruments) to spatially modulate the light intensity. The DMD is imaged onto the target tissue or sample, resulting in programmable spatial frequency patterns that are projected over a $22 \times 17 \mathrm{~mm}^{2}$ field of view.

Collection optics image a 1-mm-diameter spot from the center of the field of view onto the distal end of a $1000-\mu \mathrm{m}$ core fiber. The diffusely reflected light collected by this detector fiber is delivered to the entrance slit of a tunable grating Oriel 


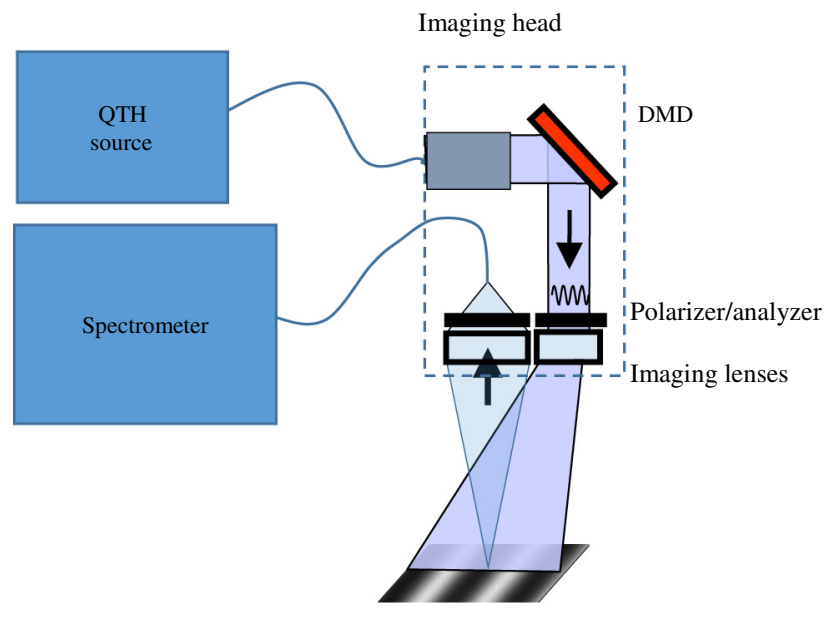

Fig. 4 A schematic diagram of the SFDS system. ${ }^{35}$

spectrograph (model no. 77480). A 16-bit, TEC-controlled CCD (Instaspec IV, Oriel) was used to detect the chromatically dispersed light. Additionally, a crossed, 2-in., wire grid polarizer/ analyzer pair is placed in the instrument to reject specularly reflected light from reaching the spectrometer. In this system, the polarizer is positioned after the projection optics, whereas the analyzer is positioned between the collection optics and the detector fiber.

Both DMD and CCD were computer controlled through a LABVIEW platform, allowing for automated data acquisition over a specified number of spatial frequencies. An autoexposure subroutine was developed to optimize the dynamic range of the CCD specific to the amount of diffusely reflected light collected from sample of interest. Through this approach, acquisition times of this particular instrument ranged from 0.1 to $0.6 \mathrm{~s} /$ pattern projected, depending on the optical properties of the sample of interest. In terms of skin tissue, this brackets the range of acquisition times needed to collect light with acceptable signal-to-noise ratio from relatively highly reflecting fair skin to highly absorbing pigment nevi.

\subsubsection{SFDS data processing}

Currently, seven spatial frequencies are acquired, ranging from 0 to $0.35 \mathrm{~mm}^{-1}$. This range was selected based on experience and the results of layered phantom investigations to differentiate spatial frequency-dependent absorption contrast from that of reduced scattering across a range of optical properties anticipated from both pigmented lesions and normal skin. Calibration measurements were also collected from a turbid reflectance standard to correct the demodulated spectra for instrumentation artifacts. Spatial frequency-dependent tissue reflectance at individual wavelengths is then fitted to homogeneous Monte Carlo models to produce unique pairs of absorption and reduced scattering coefficients. It is important to note that this method for determining optical properties is still dependent on a homogeneous model, yet these results can be exploited in such a way as to yield two-layer results, as the depth sensitivity changes across the broad range of wavelengths employed in this system. ${ }^{35}$

\subsubsection{Layer-model interpretation of SFDS data}

Based on the differential spectral response in skin from visible and NIR wavelengths, we have developed a method to estimate both the mean thickness distribution and depth-specific concentration of melanin using the optical properties determined by SFDS that were described in Sec. 2.2.2. ${ }^{35}$ This two-layer model approach makes no assumption of the thickness of tissue where melanin resides. Instead, through SFDS, this approach exploits the fact that the mean penetration depth of visible light interrogates only tenths of millimeters into skin, whereas NIR light can interrogate millimeters into tissue. Since the depth of penetration can be modeled as a function of the tissue absorption and reduced scattering properties, ${ }^{36,37}$ SFDS is capable of estimating the mean, site-specific depth penetration of light over visible and NIR spectral regimes, respectively. Fitting for chromophores in these two regions will produce concentrations of melanin and hemoglobin species relative to the entire volume interrogated. Finally, assuming that melanin and hemoglobin are mostly confined within upper and lower layer structures, respectively, the differential determinations of melanin concentration by visible and NIR regimes can be used to estimate the layer thickness in which melanin must reside to satisfy its respective concentration "detected per volume interrogated" in each regime. This model then can produce an estimate of (1) the depth distribution of melanin from the surface of skin (i.e., melanin distribution thickness), (2) the concentration of melanin within that depth distribution, and (3) melanin-corrected hemoglobin concentrations.

\subsection{High-Frequency Ultrasound}

Measurements from a high-frequency ultrasound (Epi-scan I200, Longport Inc.) were also recorded across the identified pigmented nevi and adjacent normal skin tissue. Although melanin does not provide a source of contrast in ultrasound imaging, it is possible to identify the dermal-epidermal junction with highfrequency ultrasound. ${ }^{38}$ Under the constraints of this study protocol, only clinically benign nevi are considered since, in most cases, the melanin in these moles remain confined within the epidermis (exceptions include benign dermal or combined nevi). Here, the ultrasound images can be used to measure the epidermal thickness over both nevi and adjacent tissue as a proxy to the melanin distribution thickness provided by the two-layer SFDS technique. The ultrasound-based thickness estimates can then be used as an independent measure for comparison to those provided by SFDS; however, it is worth noting that the accuracy of these ultrasound measurements is limited to $\sim \pm 20 \mu \mathrm{m}$.

\subsection{Human Data}

Twenty subjects of skin types I or II with clinically benign nevi were imaged under UCI IRB approved protocol \#2015-1791. Melanin and hemoglobin concentrations of nevi and surrounding normal skin for each of the 20 subjects were measured by SkinSpect and SFDS. SkinSpect results were compared to melanin and hemoglobin concentrations obtained with the SFDS approach.

\section{Results and Discussion}

Figures 5(a) and 5(b) show parallel and cross-polarized color images for 20 subjects with a clinically benign appearing nevus, who, following dermatologist clinical assessment of a nevus of interest, were asked to participate in this study. These images are produced by combining SkinSpect images captured under red-, green-, and blue-polarized illumination and paralleland cross-polarized image capture. For this study, we intentionally imaged nevi from different locations on the body and from 


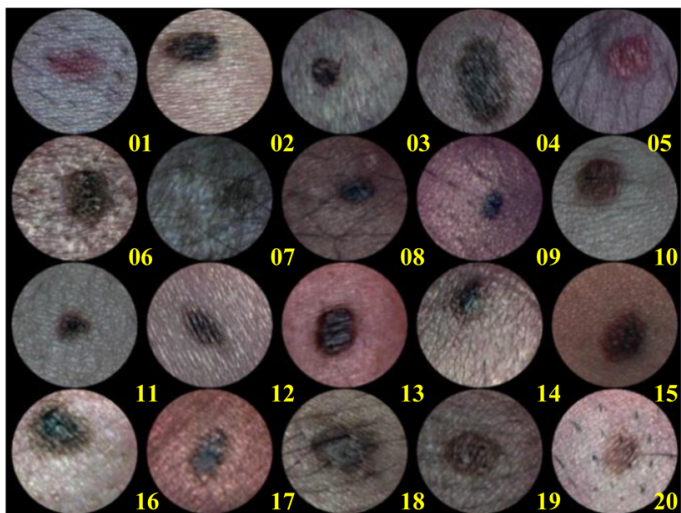

(a)

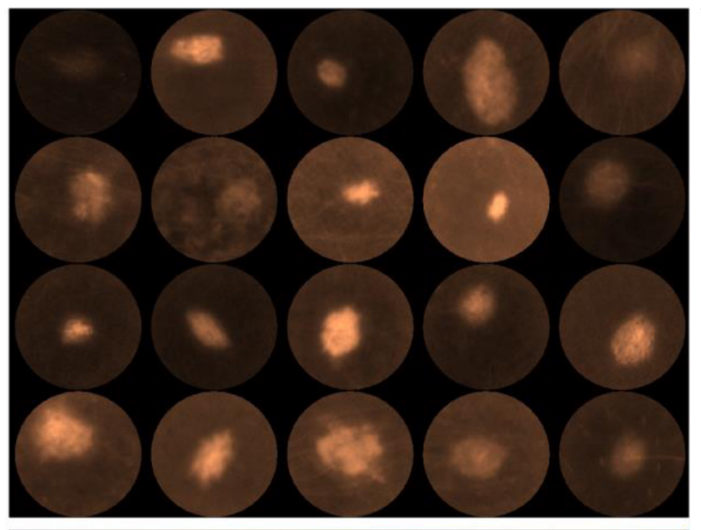

0.4

(c)

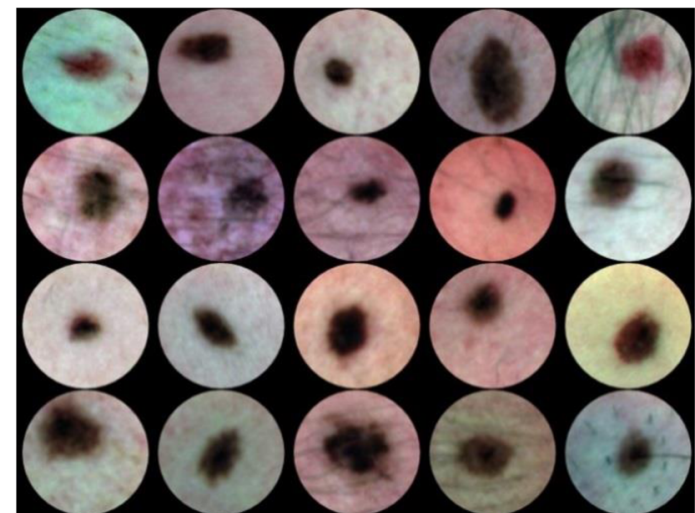

(b)

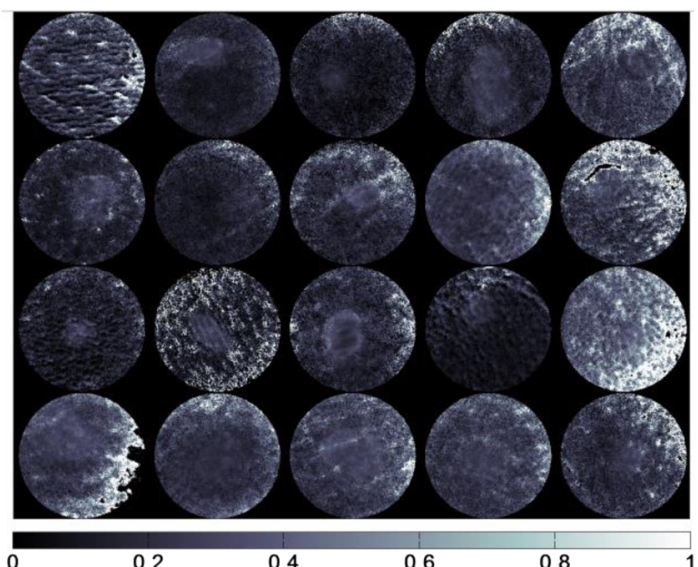

(d)

Fig. 5 Skin lesion analysis by (a) RGB color image at parallel polarization, (b) RGB color image at perpendicular polarization, (c) total melanin concentration map, and (d) deep melanin concentration map.

Fitzpatrick skin type I and II subjects of either sex and a range of ethnicities. Other skin types were not excluded but were not available in the clinic when data were collected. Figure 5(c) shows the estimated relative total melanin concentration map created by measuring the attenuation spectra (cross-polarized) slope between 615 and $680 \mathrm{~nm}$ at all pixels from the image cube as described in Sec. 2.1.2. Figure 5(d) shows an estimated relative deep melanin concentration map created by measuring the Apol attenuation spectra slope between 615 and $680 \mathrm{~nm}$. One feature we can see in deep melanin maps is bright spots of high melanin concentrations that have the same spatial coordinates as hair follicles that are visible in the RGB color pictures [Figs. 5(a) and 5(b)]. Deep melanin is estimated using a technique [Eqs. (2) and (3)] that is dependent on how polarized light propagates in skin. The parallel polarization images contain photons reflected back from both superficial and deeper layers. The cross-polarization images contain photons that have been scattered enough times to have their polarization shifted by $90 \mathrm{deg}$. The absorption changes for these photons are predominantly from deeper layers of tissue. While we can mathematically remove the superficial absorption using the Apol function, the superficial absorption may not map exactly to the epidermal-dermal junction because this boundary is not at a consistent depth, and the Apol function may not remove all the epidermal absorption. The residual deep melanin that has the same spatial coordinates as the nevus may be a result of dermal melanin or deeper epidermal melanin, or may be incompletely corrected epidermal melanin. By identifying the amount of deep melanin measured in benign nevi, we can identify a threshold of deep melanin that can be expected. Higher or abnormally distributed deep melanin can indicate melanocyte invasion (larger Breslow thickness), providing the dermatologist with a quantitative diagnostic indicator. Although in this study we were limited to benign nevi, we will be extending the SkinSpect study to subjects with suspected melanoma. Figure 6 shows maps of the relative total hemoglobin created without using melanin attenuation crosstalk correction described in Sec. 2.1.2. These relative total hemoglobin estimation maps manifest misestimations of total hemoglobin. The misestimation becomes very obvious when comparing hemoglobin and melanin maps. In the highly pigmented region, we see strong correlation (overestimation) between hemoglobin values and the presence of melanin that is not biologically plausible because in normal nevi melanin is mostly confined to the epidermal layer. Figure 6 shows the relative total hemoglobin maps after melanin attenuation crosstalk correction. Each $\mathrm{Hb}$ map is individually normalized to better illustrate the hemoglobin variations within the field of view. The $\mathrm{Hb}$ intensity maps are normalized such that $1 \%$ of data are saturated at low and high intensities. As shown in Fig. 6, in both pigmented and normal skin areas, we have seen a decrease of total hemoglobin. This is due to removing the melanin absorption from total attenuation for all the fields of view 


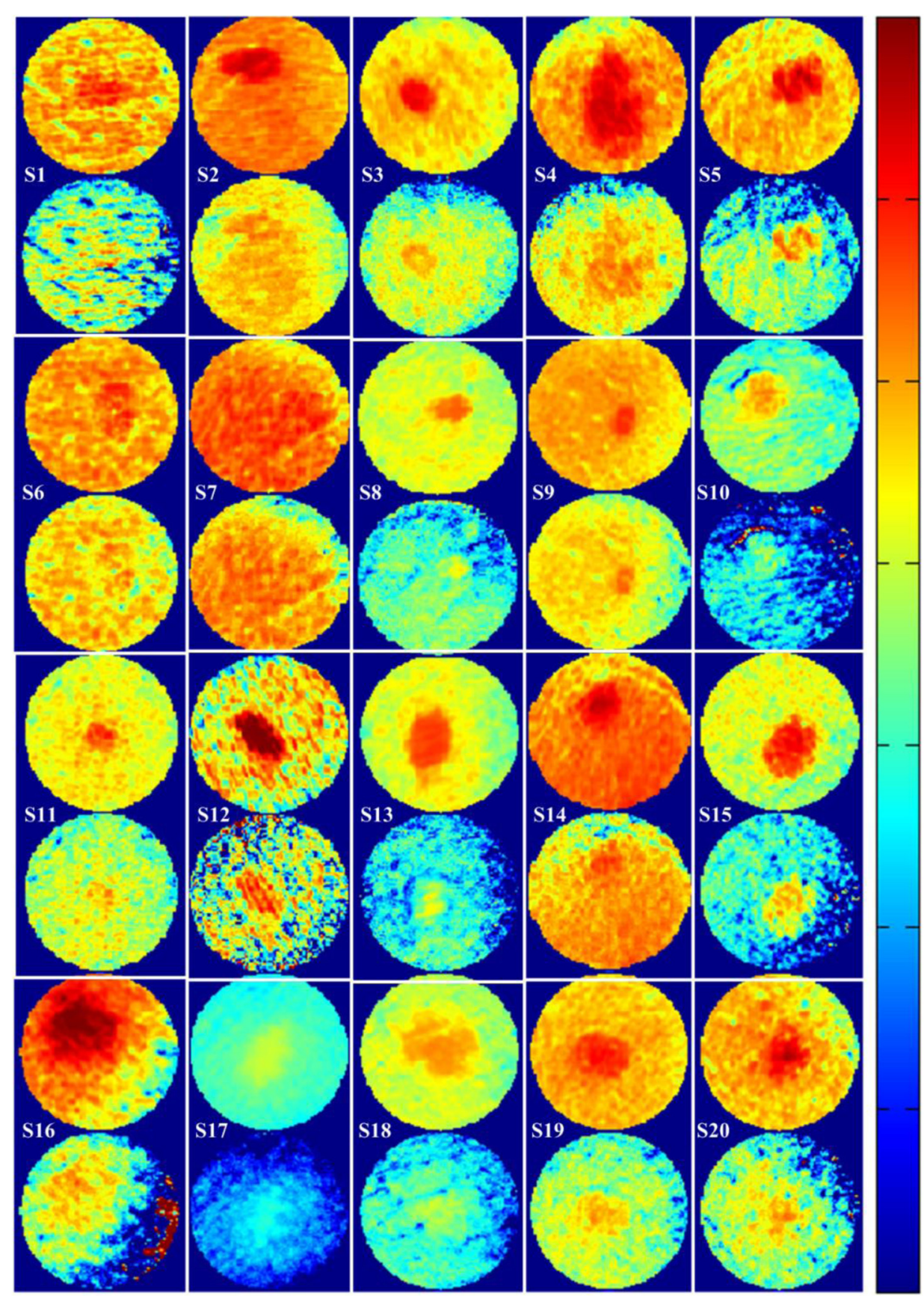

Fig. 6 Skin lesion analysis by total $\mathrm{Hb}$ before melanin attenuation correction (top) and total $\mathrm{Hb}$ after melanin attenuation correction (bottom). The images in total $\mathrm{Hb}$ maps are contrast enhanced for each individual subject to improve the visibility.

while the hemoglobin distribution of pigmented areas and surrounding normal skin become closer to each other.

The Hb maps in Fig. 6 show a significantly reduced correlation to the relative melanin maps, showing effective correction of crosstalk and producing maps that are much closer to being biologically plausible. As shown in the total hemoglobin maps, the degree of correction varies between different nevi; in some cases, melanin influence is completely removed and in some cases, there is still visible melanin attenuation on the total hemoglobin maps.

Figures 7(a) and 7(b) show scatterplots of normalized total melanin and total hemoglobin values for subject \#1 and subject \#19 before and after melanin attenuation crosstalk correction.
The measured regions of interests are selected from the center of the nevus and the adjacent normal tissue. In these subjects, after melanin correction, the estimated hemoglobin values show much less correlation with the total melanin values since the scatterplot become much less clustered in the $Y$-axis. However, as shown in Figs. 7(c) and 7(d), the scatterplots of normalized total melanin and total hemoglobin values for subject \#19 still show clustering in the $Y$-axis around the nevus measurements where the melanin correction was less effective compared to subject \#1. The lower correlation between melanin and total hemoglobin shown both in the images and in the scatterplots validates SkinSpect's ability to minimize the effect of melanin and hemoglobin attenuation crosstalk. 

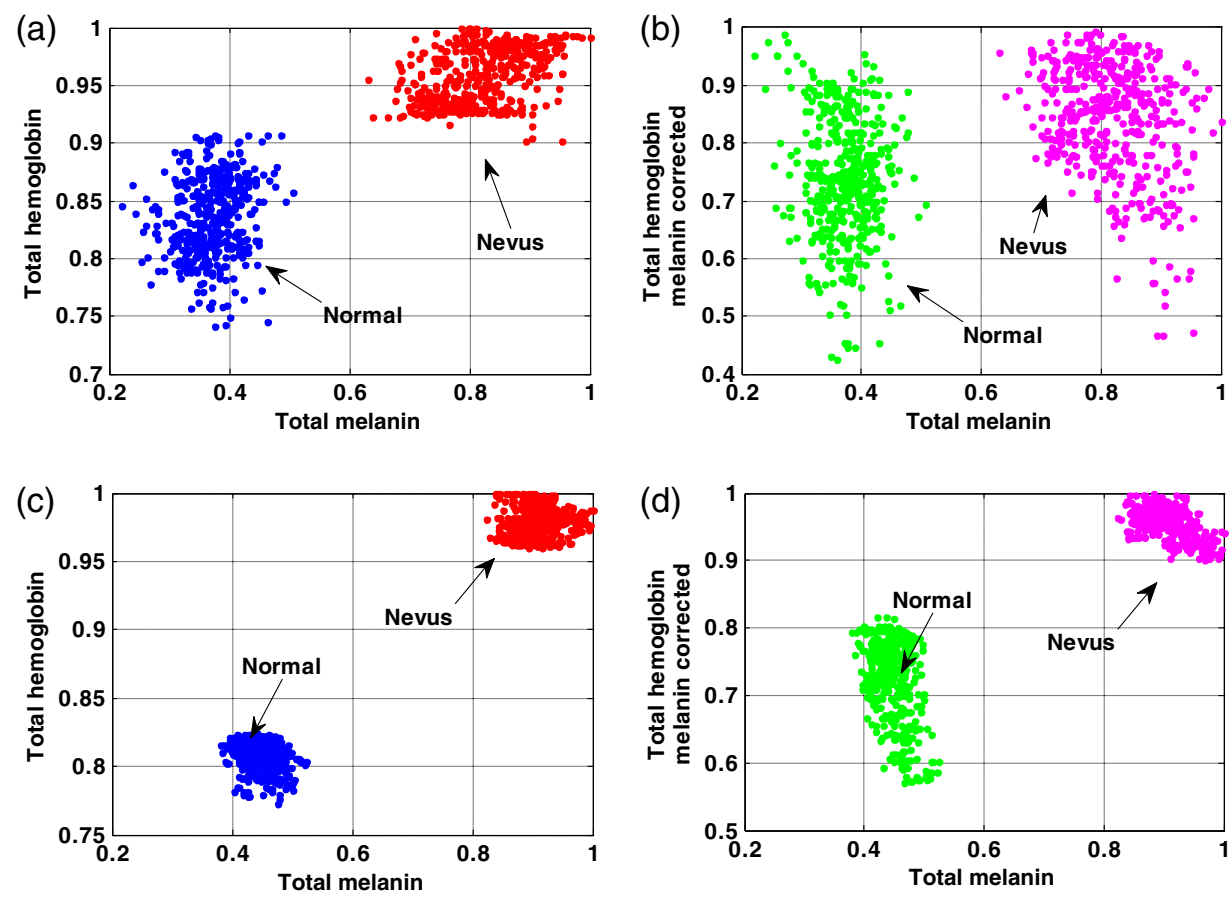

Fig. 7 Normalized total melanin and total $\mathrm{Hb}$ scatterplot in subject \#1 (a) before correcting melanin- $\mathrm{Hb}$ crosstalk and (b) crosstalk corrected. Normalized total melanin and total Hb scatterplot in subject \#19 (c) before correcting melanin- $\mathrm{Hb}$ crosstalk and (d) crosstalk corrected.

We also compared melanin and hemoglobin correlation for measurements made with SFDS. The correlation analysis for total melanin and hemoglobin concentrations measured by the SFDS system is presented in Fig. 8(a). As expected, there is very little correlation between melanin and hemoglobin values $(R=0.13)$. Figure 8 (b) compares the estimate of total melanin for the nevus and adjacent normal regions by SkinSpect and the SFDS system. The measurement sampling regions are somewhat different since we are comparing an imaging system and a point measurement system; also, for some subjects, repositioning was required. For SkinSpect, we have averaged the melanin concentration of a $21 \times 21$-pixel region of interest (ROI; $0.525 \times 0.525 \mathrm{~mm}$ ) at the center of each nevus when we compare it to SFDS melanin estimation results. Melanin estimation by the two systems shows a moderate correlation value $(R=0.63)$. It was found that two of the outlying data points correlated with smaller nevi (minimum diameter $<1.5 \mathrm{~mm}$ ). Considering that the SFDS system integrates diffuse reflectance only at a single point over a 1-mm-diameter area at the surface of the tissue, it is likely that lesions smaller than $1.5 \mathrm{~mm}$ will present challenges for the current SFDS system to accurately locate and ensure that the detected optical signal was all collected from the nevus itself and did not include adjacent tissue. By identifying which subset of nevi measured by SFDS may contain both nevi and adjacent tissue by size of nevus alone, the remaining population is reduced to $n=18$ (nevi) and $n=20$ (normal skin), but the correlation between the two optical modalities increases to $R=0.69$.

From Fig. 8(b), these smaller nevi are labeled (subjects \#8 and \#9). These nevi account for the outliers where SFDS underestimates the melanin concentration relative to expected values, further supporting the hypothesis that these measurements are not purely derived from within the pigmented lesion but also contain a contribution of the adjacent (lighter) skin tissue.

As the accuracy of the melanin concentration values determined via SFDS is directly dependent on the technique's ability
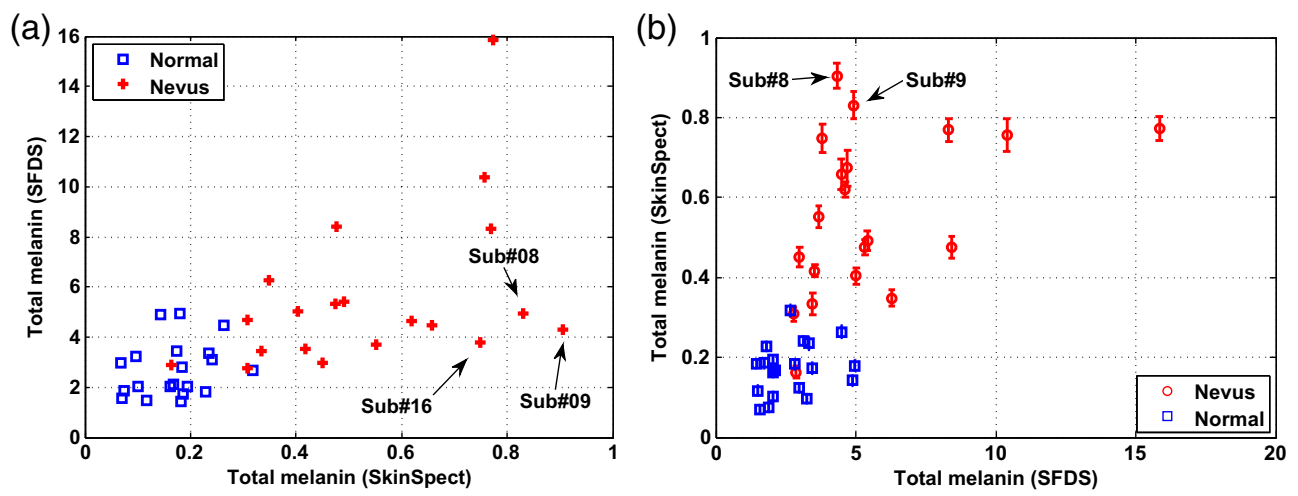

Fig. 8 (a) Melanin and total $\mathrm{Hb}$ correlation analysis estimated by SFDS system using layered model approach. (b) Melanin estimation correlation analysis between SFDS and SkinSpect. 
Table 1 Ultrasound and SFDS measurements: melanin layer thickness and subsequent depth-specific chromophore concentrations for all subjects and site locations.

\begin{tabular}{|c|c|c|c|c|}
\hline \multirow[b]{3}{*}{ Sub \# } & \multicolumn{2}{|c|}{ Ultrasound } & \multicolumn{2}{|c|}{ SFDS } \\
\hline & \multicolumn{2}{|c|}{$\begin{array}{l}\text { Epidermal } \\
\text { thickness }(\mu \mathrm{m})\end{array}$} & \multicolumn{2}{|c|}{$\begin{array}{l}\text { Epidermal } \\
\text { thickness }(\mu \mathrm{m})\end{array}$} \\
\hline & Normal & Nevus & Normal & Nevus \\
\hline 1 & 60 & 60 & 67.62 & 54.55 \\
\hline 2 & 70 & 70 & 52.58 & 80.05 \\
\hline 3 & 70 & 100 & 80.02 & 78.30 \\
\hline 4 & 70 & 70 & 94.45 & 103.7 \\
\hline 5 & 80 & 120 & 92.71 & 92.77 \\
\hline 6 & 70 & 80 & 96.19 & 99.67 \\
\hline 7 & 90 & 90 & 63.15 & 81.89 \\
\hline 8 & 70 & 70 & 91.06 & 94.23 \\
\hline 9 & 70 & 70 & 84.75 & 89.60 \\
\hline 10 & 80 & 90 & 98.07 & 96.33 \\
\hline 11 & 60 & 70 & 60.53 & 60.57 \\
\hline 12 & 80 & 80 & 75.02 & 63.21 \\
\hline 13 & 90 & 240 & 93.49 & 203.2 \\
\hline 14 & 70 & 70 & 80.55 & 87.27 \\
\hline 15 & 74 & 110 & 94.00 & 112.39 \\
\hline 16 & 70 & 90 & 94.01 & 101.21 \\
\hline 17 & 80 & 90 & 97.72 & 101.07 \\
\hline 18 & 80 & 240 & 98.97 & 124.66 \\
\hline 19 & 80 & 100 & 89.96 & 94.05 \\
\hline 20 & 90 & 90 & 100.44 & 117.96 \\
\hline
\end{tabular}

to determine the site-specific depth distribution of melanin within the skin tissue interrogated, Table 1 reports the melanin layer thickness and the subsequent depth-specific chromophore concentrations for all subjects and site locations. The epidermal thickness values for both nevus and adjacent normal skin measured by high-frequency ultrasound are also reported. In this context, the ultrasound-based thickness values provide an independent measure to compare the results determined by SFDS and its optical model. On average, SFDS reports mean thicknesses of $85 \pm 14 \mu \mathrm{m}$ for normal skin and $97 \pm 30 \mu \mathrm{m}$ for the nevi. Ultrasound reported mean thicknesses of $75 \pm 8 \mu \mathrm{m}$ for normal skin and $100 \pm 50 \mu \mathrm{m}$ for nevi. Given the previously established accuracy of the SFDS layer model to estimate melanin layer thickness to within $\pm 15 \mu \mathrm{m}$ (Ref. 31) and the ultrasound's accuracy of $\sim \pm 20 \mu \mathrm{m}$, there is strong agreement between the thickness values determined by both modalities averaged over all subjects $(R=0.742)$. There was also strong agreement between both modalities on a subject-by-subject (and site-specific) basis, where the difference between ultrasound and SFDS values was no greater than $26.8 \mu \mathrm{m}$ (max value: subject 7 , mean absolute difference overall subjects: $15.0 \mu \mathrm{m}$ ) for normal skin and $33.7 \mu \mathrm{m}$ (max value: subject 4 , mean absolute difference: $15.5 \mu \mathrm{m}$ ) for 18 of the 20 nevi.

There were two specific nevi, however, that presented discrepancies that cannot be explained by measurement error alone. Ultrasound reported substantially larger nevus thickness values for subjects 13 and 18 relative to the remaining subject population ( $\sim 2$ to $2.5 \times$ larger). These two particular subjects clinically presented raised moles relative to adjacent skin. In these cases, the epidermal thickness reported by ultrasound represented the largest thickness (from the center of the mole), although the thicknesses across the entire nevus varied from the normal skin thickness (90 and $80 \mu \mathrm{m}$, respectively) toward the center ( $240 \mu \mathrm{m}$ for both subjects). Given both the spatial resolution and accuracy of positioning the SFDS system precisely over the center of the raised mole, it is not unexpected that the layer thickness estimates from SFDS would be underestimated relative to ultrasound.

Based on the current comparison data among SkinSpect, SFDS, and the ultrasound system, each imaging technology has its own advantages and disadvantages:

SFDS advantages are to discriminate scattering from absorption, estimate total melanin and hemoglobin, estimate melanin optical thickness, discriminate melanin, and hemoglobin crosstalk, whereas the disadvantage is that the system is limited to point detection (lack of spatial resolution).

SkinSpect advantages are to estimate total melanin, deep melanin, and hemoglobin; discriminate melanin and hemoglobin crosstalk; possibly provide morphological information with spatially resolved measurements; and fast acquisition at $\sim 7 \mathrm{~s}$, processing time compared to $20 \mathrm{~s}$ using GPU processing, whereas the disadvantage of this technology is that it cannot extract melanin optical depth yet (lack of axial resolution).

Ultrasound offers high spatial resolution in depth (sub-20 microns), compactness, and a clinic-friendly footprint, while the disadvantages are no functional information regarding oxy- and deoxy-hemoglobin concentration and no information specifically related to melanin.

\section{Conclusion}

Advanced dermoscopy with molecular mapping features can be a diagnostic aid for dermatologists; however, many new approaches produce biologically implausible results, especially in melanocytic nevi where melanin absorption interferes with hemodynamics measurement. We have shown how melanin attenuation crosstalk affects hemodynamics measurement and how it is possible to substantially correct the crosstalk. We use correlation of melanin and total hemoglobin measurements to show the magnitude of crosstalk. High correlations of melanin and hemoglobin measurements indicate substantial crosstalk. SkinSpect can reduce the correlation from $R=0.63$ to $R=0.07$.

The low correlation between melanin and total $\mathrm{Hb}$ shown in all 20 subjects (both nevi and normal regions) validates the SkinSpect algorithm with melanin-hemoglobin absorption crosstalk reduced to those that are biologically plausible for the tissue examined. The SFDS system also demonstrated low correlation of melanin with hemoglobin $(R=0.13)$. Although a different method of measurement and optical model was used to disentangle the crosstalk between melanin and hemoglobin, 
the results are similar (low correlation of melanin and hemoglobin) to what we found with SkinSpect. These advances in accurate measurement of hemodynamics in the presence of high melanin absorption can lead to more biologically plausible advanced dermoscopic tools. We will further assess these approaches in the future on patients with lesions suspicious for melanoma.

\section{Acknowledgments}

Spectral Molecular Imaging, Inc. (D.L. Farkas, PI) acknowledges support from the US Department of Health and Human Services (under the Qualifying Therapeutic Discovery Program of the Patent Protection and Affordable Care Act of 2010), and the National Institutes of Health (under NCI SBIR Grant No. 1R44CA183169-01A1). Dr. Durkin and Dr. Saager thankfully recognize support from the Arnold \& Mabel Beckman Foundation and the NIH, including No. P41EB015890 (A Biomedical Technology Resource) from NIBIB. R.B.S., T.M., K.M.K., and A.J.D. declare no competing interests. F.V. and N.B. are Research Scientists for SMI. N.M. is a consultant for SMI. D.L.F. is CEO and Chairman of SMI. SMI is a for-profit corporate entity that intends to use the multimode dermoscope and approach described in this paper for early diagnosis of melanoma, and commercialize the device, after securing regulatory approval for it.

\section{References}

1. "SEER database," http://seer.cancer.gov/statfacts/html/melan.html (09 March 2016).

2. "Cancer facts and figures 2016," American Cancer Society, http://www. cancer.org/acs/groups/content/@ research/documents/document/acspc047079.pdf (17 March 2016).

3. G. P. Guy et al., "Prevalence and costs of skin cancer treatment in the US, 2002-2006 and 2007-2011," Am. J. Prevent. Med. 48(2), 183-187 (2015).

4. C. M. Balch et al., "The revised melanoma staging system and the impact of mitotic rate," Melanoma Lett. 28(3) (2010).

5. A. Mullard, "FDA approves first immunotherapy combo," Nat. Rev. Drug Discovery 14(11), 739-739 (2015).

6. D. Kapsokalyvas et al., "Spectral morphological analysis of skin lesions with a polarization multispectral dermoscope," Opt. Express 21(4), 4826-4840 (2013).

7. S. Vyas, A. Banerjee, and P. Burlina, "Estimating physiological skin parameters from hyperspectral signatures," J. Biomed. Opt. 18(5), 057008 (2013).

8. D. Yudovsky and L. Pilon, "Retrieving skin properties from in vivo spectral reflectance measurements," J. Biophotonics 4(5), 305-314 (2011).

9. N. MacKinnon et al., "Melanoma detection using smartphone and multimode hyperspectral imaging," Proc. SPIE 9711, 971117 (2016).

10. G. Argenziano et al., "Epiluminescence microscopy for the diagnosis of doubtful melanocytic skin lesions: comparison of the $\mathrm{ABCD}$ rule of dermatoscopy and a new 7-point checklist based on pattern analysis," Arch. Dermatol. 134(12), 1563-1570 (1998).

11. F. Vasefi et al., "Polarization-sensitive hyperspectral imaging in vivo: a multimode dermoscope for skin analysis," Sci. Rep. 4(4924), 1-10 (2014).

12. F. Vasefi, N. B. MacKinnon, and D. L. Farkas, "Toward in-vivo diagnosis of skin cancer using multimode imaging dermoscopy: (II) molecular mapping of highly pigmented lesions," Proc. SPIE 8947, 89470J (2014).

13. J. Marcoval et al., "Vascular density and survival in cutaneous melanoma," Br. J. Dermatol. 134(4), 809-810 (1996).

14. J. Marcoval et al., "Angiogenesis and malignant melanoma. Angiogenesis is related to the development of vertical (tumorigenic) growth phase," J. Cutaneous Pathol. 24(4), 212-218 (1997).

15. O. Straume, H. B. Salvesen, and L. A. Akslen, "Angiogenesis is prognostically important in vertical growth phase melanomas," Int. J. Oncol. 15, 595-599 (1999).
16. E. N. Ioannidis, K. Aroni, and N. Kavantzas, "Assessment of vascularity in common Blue Nevi, small/medium congenital nevocellular, common and dysplastic acquired melanocytic nevi and melanomas: a comparative study," Am. J. Dermatopathol. 36(3), 217-222 (2014).

17. A. L. Breslow, "Thickness, cross-sectional areas and depth of invasion in the prognosis of cutaneous melanoma," Ann. Surg. 172(5), 902-908 (1970).

18. A. L. Breslow, "Tumor thickness, level of invasion and node dissection in stage I cutaneous melanoma," Ann. Surg. 182(5), 572-575 (1975).

19. M. Kashani-Sabet et al., "Tumor vascularity in the prognostic assessment of primary cutaneous melanoma," J. Clin. Oncol. 20(7) 18261831 (2002).

20. J. M. Schmitt and G. Kumar, "Turbulent nature of refractive-index variations in biological tissue," Opt. Lett. 21(16), 1310-1312 (1996).

21. R. R. Anderson and J. A. Parrish, "The optics of human skin," J. Invest. Dermatol. 77(1), 13-19 (1981).

22. J. Sandby-Moller, T. Poulsen, and H. C. Wulf, "Epidermal thickness at different body sites: relationship to age, gender, pigmentation, blood content, skin type and smoking habits," Acta Derm.-Venereol. 83(6), 410-413 (2003).

23. S.L. Jacques, "Origins of tissue optical properties in the UVA, visible, and NIR regions," in OSA TOPS on Advances in Optical Imaging and Photon Migration, Vol. 2, pp. 364-369 (1996).

24. J. R. Weber, D. J. Cuccia, and B. J. Tromberg, "Modulated imaging in layered media," in IEEE Engineering Medicine Biology Society Int. Conf. Proc., pp. 6674-6676 (2006).

25. E. Angelopoulou, "Understanding the color of human skin," in Int. Society for Optics and Photonics in Photonics West 2001-Electronic Imaging, pp. 243-251 (2001).

26. A. N. Yaroslavsky et al., "Optics of blood," in Handbook of Optical Biomedical Diagnostics, V. V. Tuchin, Ed., pp. 169-216, SPIE, Bellingham, Washington (2002).

27. G. Zonios, J. Bykowski, and N. Kollias, "Skin melanin, hemoglobin, and light scattering properties can be quantitatively assessed in vivo using diffuse reflectance spectroscopy," J. Invest. Dermatol. 117(6), 1452-1457 (2001).

28. "Biophysically-based spectral model of light interaction with human skin," University of Waterloo, Canada, http://www.npsg.uwaterloo.ca/ models/biospec.php (16 June 2016).

29. T. Sarna and H. M. Swartz, "The physical properties of melanins," in The Pigmentary System, J. J. Nordlund et al., Eds., Oxford University Press, Oxford, United Kingdom (1988).

30. S. Prahl, "Optical absorption of hemoglobin," Oregon Medical Laser Center, http://omlc.org/spectra/hemoglobin/ (16 June 2016).

31. R. B. Saager et al., "In vivo measurements of cutaneous melanin across spatial scales: using multiphoton microscopy and spatial frequency domain spectroscopy," J. Biomed. Opt. 20(6), 066005 (2015).

32. T. B. Fitzpatrick, "Soleil et peau," J. de Médecine Esthétique 2, 33-34 (1975).

33. R. B. Saager et al., "In vivo isolation of the effects of melanin from underlying hemodynamics across skin types using spatial frequency domain spectroscopy," J. Biomed. Opt. 21(5), 057001 (2016).

34. G. Hoxter, "Suggested isosbestic wavelength calibration in clinical analyses," Clin. Chem. 25(1), 143-146 (1979).

35. R. B. Saager et al., "Method for depth-resolved quantitation of optical properties in layered media using spatially modulated quantitative spectroscopy," J. Biomed. Opt. 16(7), 077002 (2011).

36. S. L. Jacques and S. A. Prahl, "Modeling optical and thermal distributions in tissue during laser irradiation," Lasers Surg. Med. 6(6), 494-503 (1987).

37. S. A. Carp, A. P. Scott, and V. Venugopalan, "Radiative transport in the delta-P1 approximation: accuracy of fluence rate and optical penetration depth predictions in turbid semi-infinite media," J. Biomed. Opt. 9(3), 632-647 (2004).

38. R. K. Mlosek and S. Malinowska, "Ultrasound image of the skin, apparatus and imaging basics," J. Ultrasonogr. 13(53), 212-221 (2013).

Fartash Vasefi is a director of engineering in R\&D at SMI Inc. In particular, he is interested in commercially adoptable technologies that use multimode optical imaging systems, advanced image processing methods, and integrated micrometer to nanometer scale optical devices in biological sensing and imaging systems. His scientific contribution includes publications in more than 20 journal articles, 70 conference proceedings, and 2 book chapters. 
Nicholas MacKinnon is VP Product Development at SMI Inc. His interests and focus are on commercialization of cost-effective optical technologies. An experienced entrepreneur, involved with eight medical device startups, and was a coinventor of the VELscope, which has screened millions of patients for oral cancer. His background includes over 30 years on development of clinical and research medical devices, working in academic research with the BC Cancer Agency, and with several medical device and instrument companies.

Rolf Saager is currently a project scientist specializing in biomedical imaging and spectroscopy at the Beckman Laser Institute (BLI), University of California (UC), Irvine. He received his $\mathrm{PhD}$ in optics from the University of Rochester.

Kristen M. Kelly is a professor of dermatology and surgery at the BLI, UC, Irvine. She received her MD degree from UCLA and is an American Board of Dermatology fellow. Her research focuses on noninvasive cutaneous imaging, cutaneous vascular lesion pathogenesis, and development of dermatologic light-based therapeutics. She is a codirector of the UC Irvine Cancer Center Skin Disease Oriented Team and the current president of the American Society for Laser Medicine and Surgery.

Tyler Maly is a PGY-4 dermatology resident physician at the UC, Irvine. He received his undergraduate and medical degrees at the University of Arizona.
Nicholas Booth received his BSc degree in physics from the University of Newcastle upon Tyne, UK, in 1993, his MSc degree in surface science from Loughborough University, UK, in 1994, and his $\mathrm{PhD}$ in physics from Warwick University, UK, in 1998. He joined SMI in 2013, where he is a director of applied physics. His current interests are focused upon hyperspectral imaging in the fields of medicine and food safety.

Anthony J. Durkin is an associate professor at the BLI at the UC, Irvine. His research is focused on the development and application of optical spectroscopic and quantitative wide-field imaging techniques to characterize superficial tissues in vivo. He is a codirector of wide-field functional imaging program at BLI. He holds his PhD in biomedical engineering from the University of Texas at Austin with emphasis on biomedical optics and spectroscopy.

Daniel L. Farkas is a former Fulbright scholar, directed a National Science and Technology Center at Carnegie Mellon University that won the Smithsonian Award for Science. He was a professor of bioengineering in University of Pittsburgh and a vice-chairman for research and a professor in the Department of Surgery, CedarsSinai Medical Center. With several prestigious awards, 200+ publications, member of 11 editorial boards, chair of 32 international conferences, his focus is on translational biomedical optical imaging, in startups and academia. 